\title{
The Topology of Technology Graphs: Small World Patterns in Electronic Circuits
}

\author{
Ramon Ferrer i Cancho ${ }^{1}$, Christiaan Janssen ${ }^{1}$ and Ricard V. Solé , $^{1,2,3, *}$ \\ ${ }^{1}$ Complex Systems Research Group, FEN-UPC, Campus Nord, B4-B5, Barcelona 08034 SPAIN \\ ${ }^{2}$ Santa Fe Institute, 1399 Hyde Park Road, New Mexico 87501, USA \\ ${ }^{3}$ NASA-associated Astrobiology Institute, CSIC/INTA, Carretera del Ajalvir Km 4, Madrid
}

\begin{abstract}
Recent theoretical studies and extensive data analyses have revealed a common feature displayed by biological, social and technological networks: the presence of small world patterns. Here we analyse this problem by using several graphs obtained from one of the most common technological systems: electronic circuits. It is shown that both analogic and digital circuits exhibit SW behavior. We conjecture that the SW pattern arises from the compact design in which many elements share a small, close physical neighborhood plus the fact that the system must define a single connected component (which requires shortcuts connecting different integrated clusters). The degree distributions displayed are consistent with a conjecture concerning the sharp cutoffs associated to the presence of costly connections [Amaral et al., Proc. Natl. Acad. Sci. USA 97, 11149 (2000)] thus providing a limit case for the classes of universality of small world patterns from real, artificial networks. The consequences for circuit design are outlined.
\end{abstract}

PACS number(s):05.40.-a

\section{INTRODUCTION}

A new class of disordered networks, the so-called small-world (SW) networks [1,2], has been shown to be widespread in very different contexts, including molecular biology [3,4], neural nets [5], Internet topology [6,7], social and scientific collaboration networks [8-11], ecosystems $[12,13]$ or the human language [14]. The presence of SW patterns might provide new, unsuspected clues to the origins of complex networks and to some of their intrinsic emergent properties. These properties would include their evolvability, robustness against external fluctuations or their fragility against unexpected sources of challenge $[15,12]$. The observation that some of these systems, with a very different origin, display similar statistical features [7] allows to develop theoretical models inspired in some methods of statistical mechanics in which the details of the units are not explicitly included.

Electronic circuits can be viewed as networks in which vertices (or nodes) are electronic components (e.g. logic gates in digital circuits and resistors, capacitors, diodes and so on in analogic circuits) and connections (or edges) are wires in a broad sense. The evolution of electronic circuits underwent two fundamental events for our concerns. First, the birth of digital circuits replacing and extending the capabilities of analogic circuits. Second, integration allowing to reduce the size of electronic equipment maintaining the same functionality. As a result, the construction of larger circuits was favored. But as far as we know, no systematic analysis of the resulting topology has been performed.

Using the formalism of graph theory, any of these nets can be described in terms of a graph $\Omega$, defined as a pair: $\Omega=(W, E)$, where $W=\left\{w_{i}\right\},(i=1, \ldots, N)$ is the set of $N$ nodes and $E=\left\{\left\{w_{i}, w_{j}\right\}\right\}$ is the set of edges/connections between nodes. Here $\xi_{i j}=\left\{w_{i}, w_{j}\right\}$ indicates that there is an edge (and thus a link) between nodes $w_{i}$ and $w_{j}$. Two connected nodes are are called adjacent and the degree of a given node is the number of edges connecting it with other nodes.

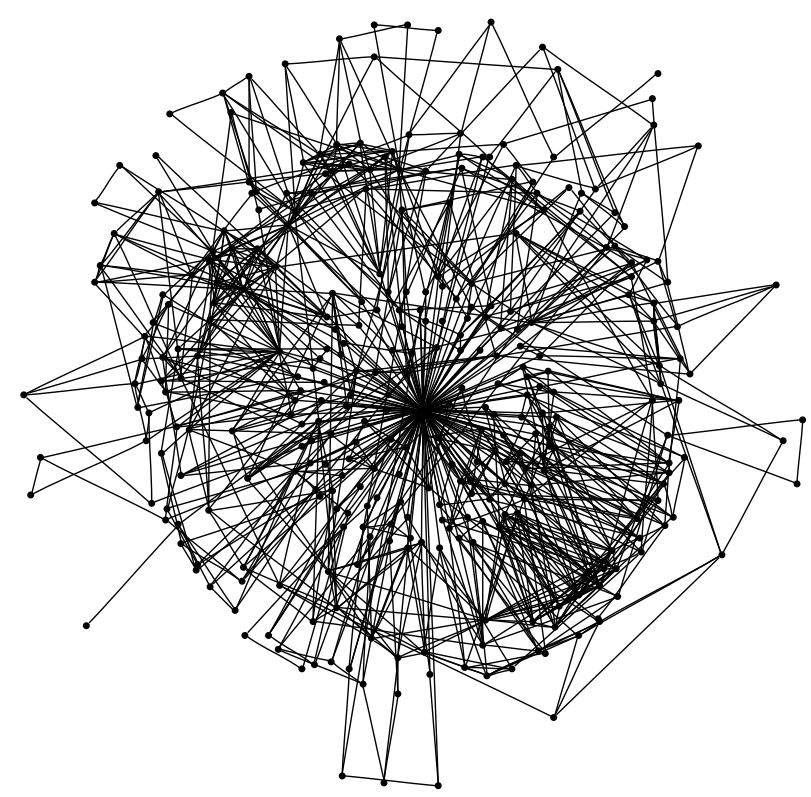

FIG. 1. The graph displayed by an analogic device (old TV circuit) in which each node represents one component (resistors, capacitors, diodes and so on). Here $N=329$ components define the graph, with an average connectivity $\langle k\rangle=5.12$. This graph has a SW structure, with: $C=0.34 \gg C^{\text {rand }}=0.019$ and $d=3.17 \approx d^{\text {rand }}=3.13$. 
The SW pattern can be detected from the analysis of two basic statistical properties: the so called clustering coefficient $C$ and the path length $d$. Let us consider the set of links $\xi_{i j}(i, j=1, \ldots, N)$, where $\xi_{i j}=1$ if a link exists and zero otherwise and that the average number of links per node is $\langle k\rangle$. Let us indicate by $\Gamma_{i}=\left\{s_{i} \mid \xi_{i j}=1\right\}$ the set of nearest neighbors of a node $w_{i} \in W$. The clustering coefficient for this node is defined as the number of connections between the components $w_{j} \in \Gamma_{i}$. By defining

$$
\mathcal{L}_{i}=\sum_{j=1}^{N} \xi_{i j}\left[\sum_{k \in \Gamma_{i} ; j<k} \xi_{j k}\right]
$$

we have: $C_{v}(i)=\mathcal{L}_{i} /\left(\begin{array}{c}\left|\Gamma_{i}\right| \\ 2\end{array}\right)$ so that the clustering coefficient is the average over $W$ :

$$
C=\frac{1}{N} \sum_{i=1}^{N} C_{v}(i)
$$

and measures the average fraction of pairs of neighbors of a node that are also neighbors of each other.

The second measure is easily defined. Given two nodes $w_{i}, w_{j} \in W$, let $d_{\text {min }}(i, j)$ the minimum path length connecting these two nodes in $\Omega$. The average path length of a given unit will be:

$$
d_{v}(i)=\frac{1}{N} \sum_{j=1}^{N} d_{\min }(i, j)
$$

and the path length is: $d=<d_{v}(i)>$.

Graphs with Small World structure are highly clustered but $d$ will be small. Random graphs (where nodes are randomly wired) are not clustered and have also short $d$ (Watts, 1999). At the other extreme, regular lattices with only nearest-neighbor connections among units, are typically clustered and exhibit long paths. A regular lattice can be transformed into a SW if a small fraction of nodes are rewired to randomly chosen nodes. Thus a small degree of disorder generates short paths (as in the random case) retaining the regular pattern (Watts and Strogatz, 1998). For random graphs, $C^{\text {rand }} \approx<k>/ N$. For SW graphs, $d$ is close to the one expected from random graphs, $d^{\text {rand }}$, with the same $\langle k\rangle$ and $C \gg C^{\text {rand }}$.

An additional property of these graphs is their degree distribution $P(k)$. It is defined as the (normalized) frequency of nodes having $k$ edges. The analysis of different real systems reveals different types of small-world network patterns [8] possibly defining a finite set of universality classes. All of them seem to share a remarkable deviation from what one would expect from a totally random graph. Three different types of distributions have been recently suggested to represent most of the observed patterns: (i) scale-free networks, in which $P(k) \approx k^{-\gamma}$; (ii) broad-scale networks, i. e. graphs with sharp cutoffs in their power-law degree distributions: $P(k) \approx k^{-\gamma} f\left(k / k^{*}\right)$, where $k^{*}$ gives the cutoff and (iii) single-scale distributions (either exponential or Gaussian). These distributions have been suggested to share some nontrivial features with other analogous systems from the theory of critical phenomena [8].

Amaral et al. [8] have recently conjectured that the shape of these distributions might result from the presence (or absence) of constraints limiting the number of links when connections are costly. In this sense, the presence of exponential decays or sharp cut-offs would be a consequence of costly wiring. Costly wiring should be specially obvious in technologic networks in which connections between elements involve hardware. In this context two different types of graphs have been analysed: the Internet $[7,16]$ and the electric power grid $[2,1,8]$. In this paper we consider a third, obvious example of a technologic network where such constraints should operate: electronic circuits.

This is a specially interesting system for three reasons: (a) it involves a graph in which efficient design relies to a large extent in connecting large groups of elements using short-range links being different, regular clusters connected through a small amount of short-cuts. In this sense, they are much closer to the Watts-Strogatz original model than any other system; (b) since technologic innovation has pushed these systems towards minimization of hardware connections, clear deviations from long-tailed distributions should be expected, according Amaral's et al. conjecture; (c) if relevant topological properties (such as the SW architecture) are present in these circuits, then future design strategies might find ways to optimize their tolerance to failure (which is very high in standard hardware devices).

The paper is organized as follows: In section II we present evidence for such SW patterns in electronic circuits as well as an analysis of their degree distributions. It is shown that the similarity between electronic circuits and ecological systems might be stronger than it has been pointed above. Such results and their consequences are outlined in Section III.

\section{RESULTS}

We have first performed a preliminary analysis of the basic features exhibited by old analogic designs, using available data [17]. These networks were used in order to test other types of hypothesis concerning the diversity of different components in a circuit and their average connectivity, to be compared with data from ecological systems. An example of the graph obtained for one of these circuits (a TV circuit) is shown [18] in figure 1 . We can see that the graph is highly non-random, as one would expect from a designed network. Some components are highly connected but most of them have 
a small degree. This graph has a SW structure, with: $C=0.34 \gg C^{\text {rand }}=0.019$ and $d=3.17 \approx d^{\text {rand }}=3.13$.

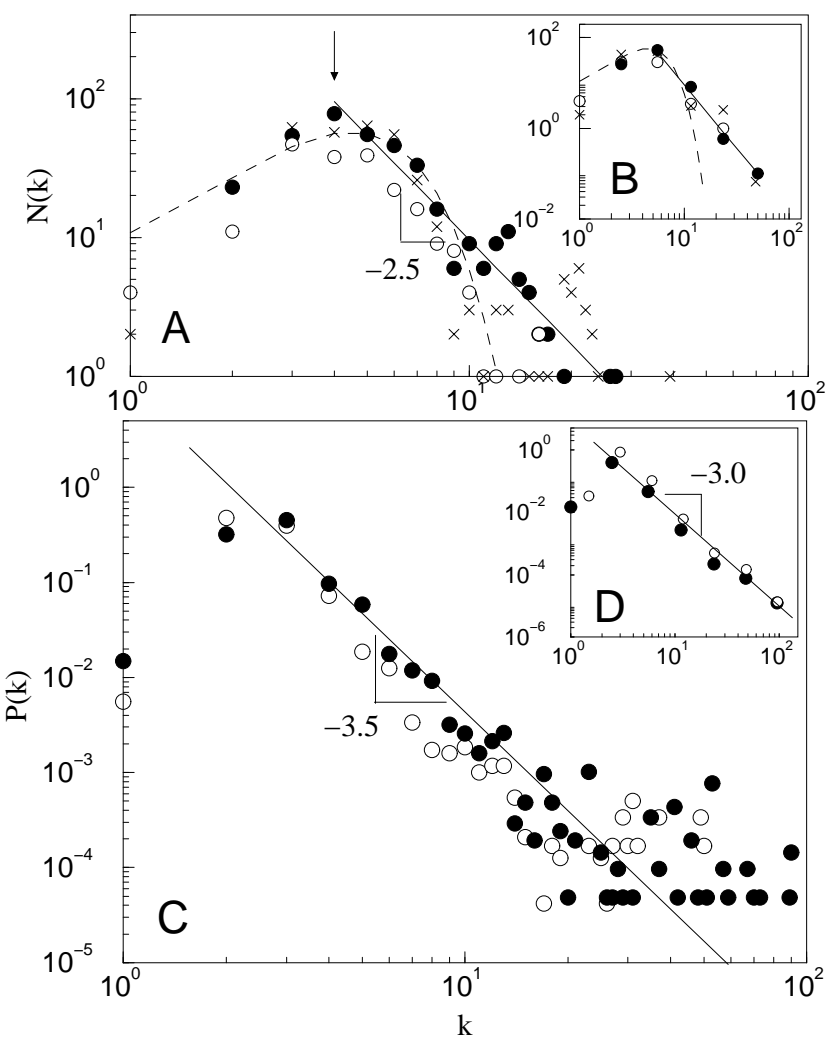

FIG. 2. (A) Degree distribution of three small-sized analogic electronic circuits (three old TV devices); here the three circuits have a size $N \approx 300$ and $\langle k\rangle \approx 5$. A characteristic maximum is observed at $k^{+}=4$, associated with a dominance of four nearest neighbors on a two-dimensional surface (thus defining a two-dimensional lattice of components). A sharp cutoff is also present at $k^{*} \approx 25-30$. In (B) the same distributions are shown using octaves. The expected distributions for a random graph with the same average connectivity is also shown (dashed line). It can be seen a clear deviation from the expected random distribution. (C) Degree distributions for two large digital circuits. Here the deviation from the random case is clear, with a tail extending up to a cutoff $k^{*} \approx 100 ;$ Inset $(\mathrm{D})$ : same as (B).

The degree distribution for the three largest networks $N \approx 350$ analysed in [17] is shown in 2(a). A characteristic maximum at $k_{c}=4$ can be seen (with an average connectivity $\langle k\rangle \approx 5$ ). This is not surprising, since a minimum of two links is typically expected (except for input/output units) and the analogic system is built on a two-dimensional substrate thus favoring topological arrangements characteristic of a two-dimensional square lattice. The fact that it can be seen a degree distribution centered around $k_{c}$ and having a sharp cut-off at $k^{*} \approx 25-30$ gives support to the Amaral's et al. conjecture concerning the limitations imposed by costly wiring. For comparison, we also show (dashed line) the expected distribution for a purely random system with the same average connectivity. It can be seen that the actual distribution strongly deviates from the random. For small $k$ it is easily understood due to the obvious limitations imposed by the circuit wiring. At larger $k$, however, it is remarkable to see that the cut off occurs at much higher values. Although these distributions are not long-tailed, we indicate the power-law fit gives an exponent $\gamma \approx 2.5$.
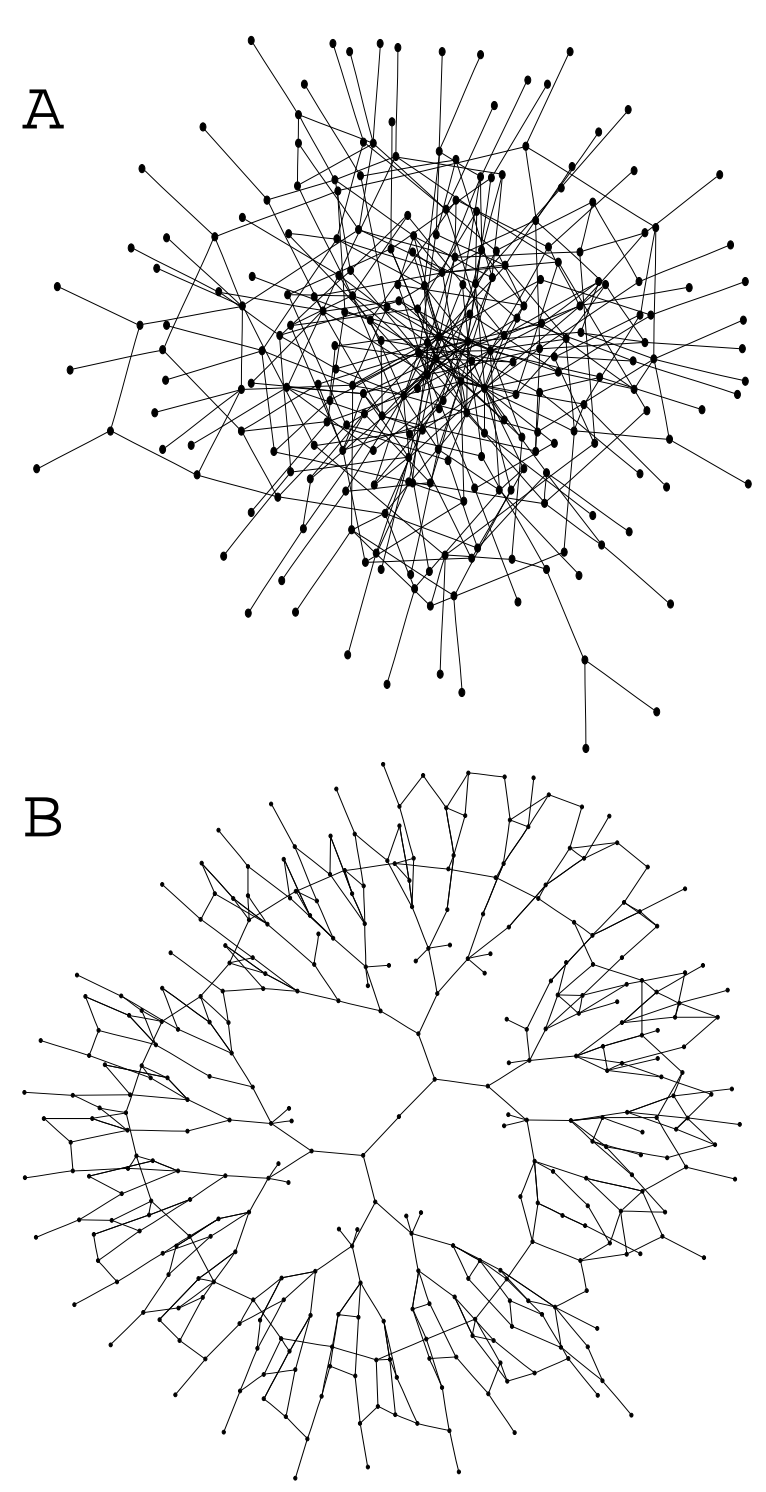

FIG. $3 . \quad$ (A) A lowly clustered logic circuit having $C=0.0013<C^{\text {rand }}=0.015$ and $d=4.33 \approx d^{\text {rand }}=4.22$. The graph has $N=236$ vertices and $\langle k\rangle=3.64$.(B) A highly clustered logic circuit having $C=0.053>C^{\text {rand }}=0.0099$ and $d=5.06 \approx d^{\text {rand }}=4.99$.

The graph has $N=320$ vertices and $\langle k\rangle=3.175$. 
Our second set of circuits provides a better understanding of how these graphs are organized in digital circuits. This set contains benchmark circuits (from the the so called ISCAS'89 and ITC'99 sets [19]). The degree distribution of two large logic circuits $N \approx 10^{4}$ is shown in 2 (c). Again, sharp cut-offs are at play, now at larger values. When looking at 2 (d) we again observe the tendency towards a power-law tail with a sharp cut-off. The estimated exponent is close to $\gamma \approx 3$. It is interesting that this value is close to the one obtained from the Albert-Barabási model and this might have interesting consequences for circuit design (see below).
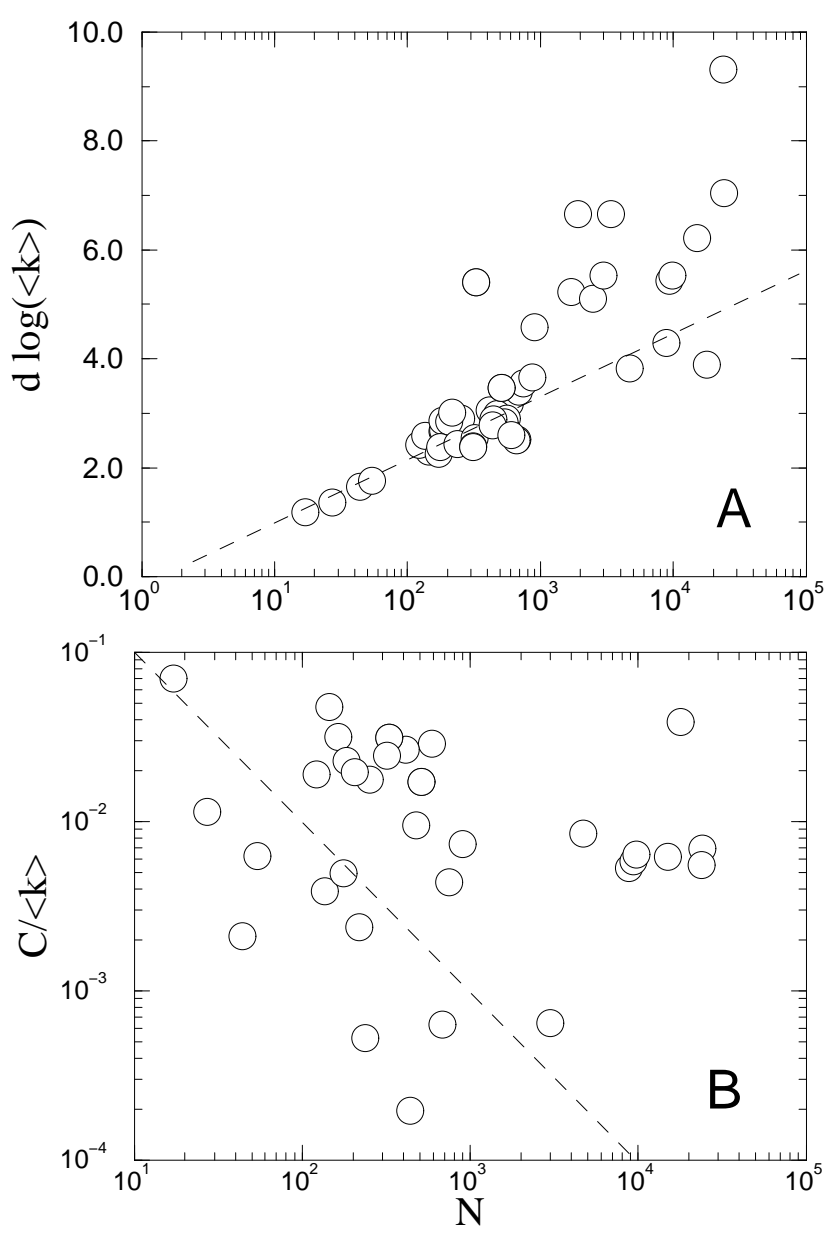

FIG. 4. Small world patterns in networks: distance (A) and clustering is (B) for the circuits in the ISCAS'89/ITC'99 are shown. Real distance is corrected by a factor of $\log \langle k\rangle$ and clustering by $\langle k\rangle$. Dashed lines correspond to the values expected for random graphs, i. e.: $C^{\text {rand }} /<k>$ and $d^{\text {rand }} \log \langle k\rangle$, respectively. It can be seen that larger circuits involve larger deviations from the random cases.

having two connections (one input and one ouput as in NOT gates and D-type flip-flops) and three connections (two inputs and one output as in AND2, OR2 gates). It is straightforward that $\langle k>\geq 2$. If components made use of the minimum number of connections (as it is expected due to optimizations in the logical design), it should be true that $2 \leq<k>\leq 3$. In contrast, the value of $\langle k\rangle$ averaged over all the circuits in the ISCAS'89/ITC'99 set is 3.65 (cal donar desviacio estandard i valor maxim i minim???). Since an input wire must receive input from only one single output (except external input), $\langle k\rangle>3$ can only be obtained by making a gate to deliver its output more than one different inputs. The question is why high values of $\langle k\rangle$ (i.e. $\langle k>\gg 4$ ) are not found. Will such values be redundant in a way that logical optimization can not tolerate? Is it necessary to go beyond $<k>=3$ for the circuit to perform a non-trivial task? Two predictions from random graph topologies will be used in order to compare them against the observed topological patterns exhibited by the benchmark circuits:

1. The clustering coefficient over the average connectivity for a random graph follows an inverse scaling law with graph size:

$$
\frac{C^{\text {rand }}}{<k>}=\frac{1}{N}
$$

2. The average path length scales as:

$$
d^{r a n d} \log (<k>) \approx \log (N)
$$

After analyzing 51 logic circuits in the ISCAS'89/ITC'99 set, 25 circuits have $C>C^{\text {rand }}$ and 26 circuits have $C<C^{\text {rand }}$, from which 17 have $C=0$. Figure 3 (a) and (b) show a circuit having $C<C^{\text {rand }}$ and another one having $C>C^{\text {rand }}$, respectively.

Figure 4 shows the values of $C /\langle k>$ and $d \log \langle k\rangle$ compared to those of $1 / N$ and $\log (N)$ for the logic circuits analyzed. It can be seen that $C / C^{\text {rand }}>1$ for most of the circuits. Values of $C / C^{\text {rand }}$ of more than one order of magnitude are achieved by the largest circuits while $d / d^{\text {rand }}$ remains in the same order of magnitude for whatever size of size of the network. There are some exceptions to $C / C^{\text {rand }}>1$ which can be attributed to the fact that the ISCAS'89/ITC'99 sets are intended as benchmarks. In this respect, a reduced number of circuits are not described at the lowest level and some others have been modified with the insertion of extra components. These two factors are likely to reduce clustering.

Nonetheless, the small-worldness of the circuits increases with the size of the circuit. From the industrial point of view, technological advance allowed circuits to grow in size. As the size increased, building circuits from scratch became unapproachable. It thus became necessary to build complex circuits using other basic circuits. From the one hand, these basic circuits have a reduced number of inputs and output wirings in comparison with 
its internal connections, so they are likely to be responsible for the high values of clustering observed. On the other hand it is known that a small number of wires interconnecting highly clustered units (according to Watt's model [2]) is enough for showing the small-world phenomenon at the high level.

\section{DISCUSSION}

Design of electronic circuits is divided into logical design and physical design [20]. The former specifies the network (components and connections) and the later specifies the precise physical realization of the system in a particular technology. Optimization is present at both stages. Automatic methods of algebraic simplification are responsible for obtaining circuits having a minimal number of components in the logic design [21] (as for optimization at the physical level, see [20]). This simplification is likely to be the origin of the low clustering attained in one half of the circuits analysed. Simplified combinatorial circuits have the form of hierarchical networks (propagating inputs to the output without backward connections), which might explain such low clustering. It has been shown that if vertices in a network are physically placed on a ring, minimizing the logic distance (the distance between vertices in the graph) and the physical distance (the Euclidean distance between vertices) leads to small-world patterns [22].

Electronic circuits constitute an example of man-made artifacts that have evolved towards non-random configurations in which minimization of both average path length and physical distance are present. Because of their particular design, standard electronic devices are highly error prone: a single failure in one component typically leads to system failure. This is not the case of biological systems, in which networks displaying highly heterogeneous degree distributions with long tails have been shown to be particularly resilient [15]. Of course the difference comes largely form the dynamical pattern of interactions among units. Redundancy and modularity can help to overcome failure of a single unit by finding appropriate pathways able to substitute the damaged unit. Is there any analogous scenario within our context?

How reliable systems can be built from unreliable components has been a very important topic since Von Neumann's [23] and McCulloch [24] pioneering work. Using formal approaches borrowed from automata theory and statistical mechanics, these authors concluded that some amount of redundancy is required in order to satisfy a number of lower bounds of system functioning. One interesting framework in which our results might help to provide new ways of generating reliable circuits is the emerging area of adaptive and evolutionary hardware $[25,26]$. A new generation of electronic devices is based on the possibility of re-configuration of the circuits wiring
[27]. Self-reconfiguration is needed to endow devices with the flexibility of in-situ challenges, adaptation to unforeseen conditions and with enhanced fault-tolerance. This is the case, for example, of planetary space missions.

The idea behind evolutionary synthesis of electronic circuits is to employ an evolutionary/genetic algorithm to control the search for a circuit (through a potentially vast parameter space) that satisfies specified objectives. The evolutionary algorithm selects a population of potential designs, coded as bit strings configurations, and downloads them to the reconfigurable chip. Evolved circuits can have some flexibility which might allow them to work safely under different sources of noise or damage. One possible constraint to be introduced into evolutionary search would be inspired in the properties displayed by biological systems displaying the SW topology. Searching for circuits with SW structure and/or longtailed distributions of links might give some new insight into the origins of the SW behavior in both natural and artificial systems. The fact that the observed degree distributions of real designs are already power laws allows to conjecture the possibility of reaching high levels of robustness against the random failure of units under an appropriate level of redundancy.

(*) Corresponding author

email: ricard@ants.upc.es, sole@santafe.edu

\section{ACKNOWLEDGMENTS}

We are grateful to Emília Gutiérrez for providing us with the analogic circuits in [17]. This work was supported by the Santa Fe Institute (RVS) and grants of the Generalitat de Catalunya (FI/2000-00393, RFC) and the CICYT (PB97-0693, RVS).

[1] D. J. Watts, Small-worlds: the dynamics of networks between order of randomness, Princeton studies in complexity (Princeton University Press, Princeton, N.J., 1999).

[2] D. J. Watts and S. H. Strogatz, Nature 393, 440 (1998).

[3] H. Jeong, B. Tombor, R. Albert, Z. N. Oltvai, and A.L.Barabási, Nature 407, 651 (2000).

[4] A. Wagner and D. Fell, Santa Fe Institute Working Paper 00-07-041 (2000).

[5] L. F. Lago-Fernández, R. Huerta, F. Corbacho, and J. A. Sigüenza, Phys. Rev. Lett. 84, 2758 (2000).

[6] L. A. Adamic, Procedings of the ECDL'99 Conference, LNCS 1696, Springer, 443 (1999).

[7] A.-L. Barabási and R. Albert, Science 286, 509 (1999).

[8] L. A. N. Amaral, A. Scala, M. Barthélémy, and H. E. Stanley, Proc. Natl. Acad. Sci. 97, 11149 (2000).

[9] M. E. J. Newman, Proc. Natl. Acad. Sci. 98, 404 (2001). 
[10] M. E. J. Newman, Phys. Rev.E. (2001), in press.

[11] M. E. J. Newman, Phys. Rev. E. (2001), in press.

[12] R. V. Solé and J. M. Montoya, Santa Fe Working Paper 00-11-060, submitted to Proc. Royal Soc. B.

[13] J. M. Montoya and R. V. Solé, Santa Fe Working Paper 00-10-059, submitted to J. Theor. Biol.

[14] R. Ferrer and R. V. Solé, Santa Fe Institute Working Paper 01-03-016 (2001), submitted to Proceedings of the Royal Society of London B.

[15] R. Albert, H. Jeong, and A.-L. Barabási, Nature 406, 378 (2000).

[16] R. M. G. Caldarelli and L. Pietronero, Europhys. Lett. 52, (2000).

[17] R. Margalef and E. Gutiérrez, The American Naturalist 121, 601 (1983).

[18] AT\&T Bell Laboratories, http://www.research.att.com/sw/tools/graphviz.

[19] Avaliable

at http: //www.cbl.ncsu.edu/CBL $L_{D}$ ocs/iscas89.html and http: //www.cad.polito.it/tools/itc99.html, respectively. Circuits containing errors or not being connected were excluded.

[20] S. Kirkpatrick, J. C. D. Gelatt, and M. P. Vecchi, Science 220, 671 (1983).
[21] E. McCluskey, Logic Design Principles (Prentice-Hall, New Jersey, 1986).

[22] N. Mathias and V. Gopal, Physical Review E 63, 1 (2001).

[23] J. von Neumann, in Automata Studies, edited by C. E. Shannon and J. McCarthy (Princeton U. Press, Princeton, N. J., 1956).

[24] W. S. McCulloch, in Self-organizing Systems, edited by M. C. Yovits and S. Cameron (Pergamon Press, New York, 1960), pp. 264-281.

[25] G. Taubes, Science 227, 1931 (1997).

[26] Evolvable Systems : From Biology to Hardware, Vol. 181 of Lecture Notes in Computer Science, edited by J. M. et al. (Springer, Berlin, 2000).

[27] An example of evolvable hardware is an adaptive configurable electronic circuit, such as a field programmable gate array (FPGA). An FPGA is an array of logic blocks (analogous to cells) placed in an infrastructure of interconnections, which are programmed at three distinct levels: the function of the logic cells, the interconnections between cells, and the inputs and outputs to the system. FPGAs exhibit online adaptation by configuring their architecture dynamically and autonomously. 